\title{
Correction to: Therapeutic drug monitoring of oral targeted antineoplastic drugs
}

\author{
Anna Mueller-Schoell ${ }^{1,2} \cdot$ Stefanie L. Groenland ${ }^{3} \cdot$ Oliver Scherf-Clavel $^{4} \cdot$ Madelé van Dyk $^{5} \cdot$ Wilhelm Huisinga $^{6}$. \\ Robin Michelet ${ }^{1} \cdot$ Ulrich Jaehde $^{7} \cdot$ Neeltje Steeghs ${ }^{3} \cdot$ Alwin D. R. Huitema $^{8,9} \cdot$ Charlotte Kloft $^{1}$ (D)
}

Published online: 15 December 2020

(C) Springer-Verlag GmbH Germany, part of Springer Nature 2020

\section{Correction to: European Journal of Clinical Pharmacology https://doi.org/10.1007/s00228-020-03014-8}

Due to an error during production, some in-text and most of the in-table citations were not correctly displayed in the original previous online version of the article and have now been corrected.

The original article has been corrected.

Publisher's note Springer Nature remains neutral with regard to jurisdictional claims in published maps and institutional affiliations.

The online version of the original article can be found at https://doi.org/ 10.1007/s00228-020-03014-8

Charlotte Kloft

charlotte.kloft@fu-berlin.de

1 Department of Clinical Pharmacy and Biochemistry, Institute of Pharmacy, Freie Universitaet Berlin, Berlin, Germany

2 Graduate Research Training Program, PharMetrX, Berlin/ Potsdam, Germany

3 Department of Clinical Pharmacology, Division of Medical Oncology, The Netherlands Cancer Institute-Antoni van Leeuwenhoek, Plesmanlaan 121, 1066

CX Amsterdam, The Netherlands
4 Institute of Pharmacy and Food Chemistry, Julius-Maximilians-Universität Würzburg, Würzburg, Germany

5 College of Medicine and Public Health, Flinders University, Adelaide, SA, Australia

6 Institute of Mathematics, University of Potsdam, Potsdam, Germany

7 Department of Clinical Pharmacy, Institute of Pharmacy, University of Bonn, Bonn, Germany

8 Department of Pharmacy \& Pharmacology, The Netherlands Cancer Institute-Antoni van Leeuwenhoek, Amsterdam, The Netherlands

9 Department of Clinical Pharmacy, University Medical Center, Utrecht University, Utrecht, The Netherlands 